\title{
CONDITIONAL PASTA
}

\author{
Erik A. van DOORN \\ Faculty of Applied Mathematics, University of Twente, P.O. Box 217, 7500 AE Enschede, The Netherlands
}

\section{G.J.K. REGTERSCHOT}

Faculty of Industrial Engineering, Eindhoven University of Technology, P.O. Box 513, 5600 MB Eindhoven, The Netherlands

Received May 1987

Revised June 1988

\begin{abstract}
Let $Y$ be a stochastic process representing the state of a system and $N$ a doubly stochastic Poisson process whose intensity varies with the state of a random environment represented by a stochastic process $X$. In this context a generalization of "Pasta" (Poisson Arrivals See Time Averages) is shcwn to be valid. Various applications of the result are given.
\end{abstract}

Poisson process * PASTA * random environment * M/G/1 queue * buffer model

\section{Introduction}

Suppose $N \equiv\{N(t), t \geqslant 0\}$ is a Poisson process of events and $Y \equiv\{y(t), t \geqslant 0\}$ a stochastic process representing the state of a system. The result known as PAsta (Poisson Arrivals See Time Averages, see Wolff [8]) then tells us that the long-run fraction of events upon the occurrence of which the system is in a particular state equals the longrun fraction of time the system is in that state, provided for every point of time the future jumps of $N$ are independent of the past of $Y$. The latter condition is called the Lack of Anticipation assumption in [8].

In this paper we consider a more general situation where $N$ is a doubly stochastic Poisson process characterized by the fact that the rate of the process varies with the state of a random environment represented by a stochastic process $X=$ $\{X(t), t \geqslant 0\}$. We shall show that under an adapted Lack of Anticipation assumption, PASTA holds conditionally on the state of $X$. That is, let $s$ be a state in the state space of $X$; then, confining attention to those events upon the occurrence of which the environment is in state $s$ and those intervals of time during which the environment is in state $s$, PASTA holds. In fact, the formal statement of our result in Section 2 is more general in that for PASTA to hold conditionally on the environment being in a particular state $s$, it is not necessary for $\boldsymbol{N}$ to be a Poisson process whenever the environment is in a state $s^{*} \neq s$. In the applications we have in mind, however, $N$ is a doubly stochastic Poisson process as described above. We shall refer to our result as Conditional PASTA.

The principle of the proof in Section 2 is very simple. We assume the existence of a Poisson process $N_{s}$ which generates the jumps of $N$ when $X$ is in state $s$, and subsequently apply PASTA iwice with respect to $N_{s}$.

A typical situation where conditional PASTA can be applied is an $M / G / 1$ queue where the arrival rate (and perhaps the service rate or the service time of an arriving customer) is determined by the current state of a background Markov chain. Another example is a reservoir which receives and releases fluid flows at rates which are modulated by an underlying Markov chain. These examples will be discussed in more detail in Section 3.

In what follows $1(E)$ denotes the indicator function of the event $E$. 


\section{The result and its proof}

Let $N \equiv\{N(t), t \geqslant 0\}, X \equiv\{X(t), t \geqslant 0\}$ and $Y \equiv\{Y(t), t \geqslant 0\}$ be stochastic processes defined on some common probability space $(\Omega, \mathscr{F}, \mathscr{P})$. The process $N$ is a counting process, that is, a process with non-decreasing, right-continuous, nonnegative integer-valued sample paths. For every $t \geqslant 0, X(t)$ and $Y(t)$ take on values in arbitrary metric spaces $S_{X}$ and $S_{Y}$, respectively, while the sample paths of $X$ and $Y$ are right-continuous and have left-hand limits a.s. (almost surely).

In what follows $s$ will be an arbitrary but fixed state in $S_{X}$ satisfying the following conditions:

(i) $\{\hat{X}(t)=s\} \in$ for cvery $t \geqslant 0$;

(ii) the limit

$\lim _{t \rightarrow \infty} \frac{1}{t} \int_{0}^{t} 1(X(u)=s) \mathrm{d} u$

exists and is positive a.s.;

(iii) There exists on the probability space $(\Omega$, $F, \infty)$ a Poisson process $N_{s} \equiv\left\{N_{s}(t), t \geqslant 0\right\}$ at rate $\lambda_{s}>0$ that generates the jumps of the counting process $N$ when $X$ is in state $s$; that is, for all $u>0$,

$1(X(u-)=s) \mathrm{d} N(u) \equiv 1(X(u-)=s) \mathrm{d} N_{s}(u)$.

The interaction between $N, X, Y$ and $N_{s}$ is not specified further except for an adapted Lack of Anticipation assumption. Namely, for every $t \geqslant 0$ the processes $\left\{N_{s}(t+u)-N_{s}(t), u \geqslant 0\right\}$ and $\{(X(u), Y(u)), 0 \leqslant u \leqslant t\}$ are assumed to be independent.

Next let $A$ be an arbitrary but fixed subset of $S_{Y}$ such that $\{Y(t) \in A\} \in \mathscr{F}$ for every $t \geqslant 0$. Finally define

$V_{s}(t)=\frac{\int_{0}^{t} 1(X(u)=s) 1(Y(u) \in A) \mathrm{d} u}{\int_{0}^{\prime} 1(X(u)=s) \mathrm{d} u}$,

the fraction of time that $Y$ is in $A$, thereby confining attention to those intervals of time in $[0, t]$ during which $X$ is in state $s$, and

$$
=\frac{\int_{0}^{t} 1(X(u-)=s) 1(Y(u-) \in A) \mathrm{d} N(u)}{\int_{0}^{t} 1(X(u-)=s) \mathrm{d} N(u)}
$$

the fraction of events upon the occurrence of which $Y$ is in $A$, thereby confining attention to those events in the interval $[0, t]$ upon the occurrence of which $X$ is in state $s$.

Remark. Taking the left-hand limits $X(u-)$ and $Y(u-)$ in (2) and (4) is essential, since we do not exclude the possibility that a jump of $N$ entails an immediate change in $X$ or $Y$.

Theorem 1. There exists a random variable $V_{s}(\infty)$ such that $V_{s}(t) \rightarrow^{a . s .} V_{s}(\infty)$ if and only if there exists a random variable $W_{s}(\infty)$ such that $W_{s}(t)^{a . s .}$ $\rightarrow W_{s}(\infty)$, and in this case $V_{s}(\infty)={ }^{a . s} \cdot W_{s}(\infty)$.

Proof. By our Lack of Anticipation assumption, $\left\{N_{s}(t+u)-N_{s}(t), u \geqslant 0\right\}$ and $\{X(u), 0 \leqslant u \leqslant t\}$ are independent for every $t \geqslant 0$, so [8, Theorem 1] and our assumption that the limit in (1) exists a.s. imply

$$
\begin{aligned}
& \lim _{t \rightarrow \infty} \frac{\int_{0}^{t} 1(X(u)=s) \mathrm{d} u}{t} \\
& \stackrel{\text { a.s. }}{=} \lim _{t \rightarrow \infty} \frac{\int_{0}^{t} 1(X(u-)=s) \mathrm{d} N_{s}(u)}{N_{s}(t)} .
\end{aligned}
$$

Next, using the adapted Lack of Anticipation assumption and [8, Theorem 1] again, we conclude

$$
\begin{aligned}
& \lim _{t \rightarrow \infty} \frac{\int_{0}^{t} 1(X(u)=s) 1(Y(u) \in A) \mathrm{d} u}{t} \\
& \stackrel{\text { a.s. }}{=} \lim _{t \rightarrow \infty} \frac{\int_{0}^{t} 1(X(u-)=s) 1(Y(u-) \in A) \mathrm{d} N_{s}(u)}{N_{s}(t)}
\end{aligned}
$$

provided one limit (and herce both limits) exists a.s. As a consequence of (5) and (6) and our assumption that the limit in (1) exists and is positive a.s. we get

$$
\begin{aligned}
& \lim _{t \rightarrow \infty} V_{s}(t) \\
& \stackrel{\text { a.s. }}{=} \lim _{t \rightarrow \infty} \frac{\int_{0}^{t} 1(X(u-)=s) 1(Y(u-) \in A) \mathrm{d} N_{s}(u)}{\int_{0}^{t} 1(X(u-)=s) \mathrm{d} N_{s}(u)},
\end{aligned}
$$

provided one limit (and hence both limits) exist a.s. Finally exploiting (2) yields the theorem.

Remark. If $S_{X} \equiv\{s\}$, so that $1(X(t)=s)=1$ for every $t \geqslant 0$, Theorem 1 reduces to [8, Theorem 1] (PAsta). 
In applications the processes $X$ and $Y$ will usually satisfy stronger conditions than required by Theorem 1 . Two such situations are described in the next two corollaries, where $T_{1}, T_{2}, \ldots$ denote the epochs at which events of $N$ occur.

Corollary 1. If the processes $\{(X(t), Y(t)), t \geqslant 0\}$ and $\left\{\left(X\left(T_{n}-\right), Y\left(T_{n}-\right)\right), n=1,2, \ldots\right\}$ are stationary and ergodic, then, under the conditions of Theorem 1, for every $t \geqslant 0$ and $n=1,2, \ldots$

$$
\begin{aligned}
& P(Y(t) \in A \mid X(t)=s) \\
& \quad=P\left(Y\left(T_{n}-\right) \in A \mid X\left(T_{n}-\right)=s\right) .
\end{aligned}
$$

Corollary 2. If the processes $\{(X(t), Y(t)), t \geqslant 0\}$ and $\left\{\left(X\left(T_{n}-\right), Y\left(T_{n}-\right)\right), n=1,2, \ldots\right\}$ are regenerative with finitc mean times between regeneration, then, under the conditions of Theorem 1 ,

$$
\begin{aligned}
& \lim _{t \rightarrow \infty} P(Y(t) \in A \mid X(t)=s) \\
& \quad=\lim _{n \rightarrow \infty} P\left(Y\left(T_{n}-\right) \in A \mid X\left(T_{n}-\right)=s\right) .
\end{aligned}
$$

The additional conditions imposed in these corollaries ensure that the limits as $t \rightarrow \infty$ of $t^{-1}$ times the numerator and denominator of (3) and $(N(t))^{-1}$ times the numerator and denominator of (4), and hence the limits as $t \rightarrow \infty$ of $V_{s}(t)$ and $W_{s}(t)$, exist and assume simple forms resulting in (8) and (9).

\section{Applications}

\subsection{Markov modulated $M / G / 1$ queues}

Regterschot and de Smit [5] (see also Regterschot [4]) study a queueing model in which a Markov chain $X=\{X(t), t \geqslant 0\}$ with finite state space $S_{X} \equiv\{1,2, \ldots, N\}$ modulates arrivals and services. More precisely, whenever $X$ is in state $i$ customers arrive at a single server queue according to a Poisson process with rate $\lambda_{i}>0$ and an arriving customer has service time distribution $G_{i}$. With $W(t)$ denoting the virtual waiting time (workload) at time $t, T_{n}$ the arrival epoch of the $n$-th customer and $W_{n}$ its waiting time, it is shown in [5] by explicit calculation that for a stable system

$$
\begin{aligned}
& \lim _{t \rightarrow \infty} P(W(t) \leqslant x \mid X(t)=i) \\
& \quad=\lim _{n \rightarrow \infty} P\left(W_{n} \leqslant x \mid X\left(T_{n}-\right)=i\right)
\end{aligned}
$$

for ali $x \leqslant 0$ and $i \in S_{x}$. A similar equality is established, again by explicit calculation of both sides, for the queue length distribution in continuous time and at arrival epochs. Evidently, defining $N(t)$ as the number of arrivals in $[0, t]$, both equalities follow directly from our generalization of PASTA, specifically from Corollary 2 by virtue of the regenerative character of the process at hand (see [5]).

A special case of the above model where $N=2$ and $G_{1}=G_{2}$ is studied by van Hoorn and Seelen [7]. There, the validity of (10) and the analogous equality for queue length distributions is established by an argument based on the regenerative structure of the model.

A related model is the $M / M / 1$ queue where both arrival and service rate are modulated by a finite Markov chain $X$. Neuts [3] studies this model and establishes by explicit calculation the equality of conditional queue length distributions in continuous time and at epochs where the Markov chain makes transitions. Again this result is implied by Conditional Pasta, if we define $N(t)$ as the number of state changes of $X$ in the interval $[0, t]$.

\subsection{A buffer with Markov modulated input / output}

The buffer models studied by Anick et al. [1], Gaver and Lehoczky [2], and van Doorn et al. [6] all fit in with the following general setting (see [4]). Let $X \equiv\{X(t), t \geqslant 0\}$ be a Markov chain with finite state space $S_{X}=\{0,1, \ldots, N\}$ and generator $Q=\left(q_{i j}\right)$, which modulates the rate at which a fluid flow is received or released by a reservoir with infinite capacity. Concretely, whenever $X$ is in state $i$ the net flow rate of fluid into the reservoir is some constant $r_{i} \neq 0$, with the restriction that the content of the reservoir cannot decrease when the reservoir is empty. With $C(t)$ denoting the content of the reservoir at time $t$, the papers cited above study the distribution of $C(t)$ as $t$ goes to infinite. It may be of interest, however, to know also the limit distribution of the content of the reservoir at epochs where the underlying Markov chain makes transitions of a particular type, for instance at epochs where the net input rate of fluid changes from positive to negative since these epochs mark the end of a buildup period. Conditional PASTA enables us to obtain such distributions from the continuous-time 
distribution. Namely, let $N(t), t \geqslant 0$, denote the numbers of jumps of the Markov chain $X$ in the interval $[0, t]$. (Note that whenever $X$ is in state $i$ the process $N \equiv\{N(t), t \geqslant 0\}$ is a Poisson process at rate $q_{i} \equiv-q_{i i}$.) Then, with $T_{n}, n=1,2, \ldots$, denoting the epoch at which $N$ jumps to level $n$, the processes $\{(X(t), C(t)), t \geqslant 0\}$ and $\left\{X\left(T_{n}-\right)\right.$, $\left.C\left(T_{n}-\right), n=1,2, \ldots\right\}$ are regenerative (see [4]), so Corollary 2 yields

$$
\begin{aligned}
& \lim _{t \rightarrow \infty} P(C(t) \leqslant x \mid X(t)=i) \\
& \quad=\lim _{n \rightarrow \infty} P\left(C\left(T_{n}-\right) \leqslant x \mid X\left(T_{n}-\right)=i\right)
\end{aligned}
$$

for all $x \geqslant 0$ and $i \in S_{X}$, provided the mean times between regenerations is finite. With this information the required distributions can easily be determined, see [6].

\section{References}

[1] D Anick, D. Mitra and M.M. Sondhi, "Stochastic theory of a data-handling system with multiple sources", Bell System Tech. J. 61, 1871-1894 (1982).
[2] D.P. Gaver and J.P. Lehoczky, "Channels that cooperatively service a data stream and voice messages", IEEE Trans. Comm. 30, 1153-1162 (1982).

[3] M.F. Neuts, "Further results on the $M / M / 1$ queue with randomly varying rates", Opsearch 15, 158-168 (1978).

[4] G.J.K. Regterschot, "Wiener-Hopf factorization techniques in queueing models", Ph. D. Thesis, University of Twente, Enschede, The Netherlands, 1987.

[5] G.J.K. Regterschot and J.H.A. de Smit, "The queue M/G/1 with Markov modulated arrivals and services", Math. Oper. Res. 11, 465-483 (1986).

[6] E.A. van Doorn, A.A. Jagers and J.S.J. de Wit, "A fluid reservoir regulated by a birth-death process", Comm. Statist. Stochastic Mowels, to appear.

[7] M.H. van Doorn and L.P. Seelen, “The SPP/G/1 queue: a single server queue with a switched Poisson process as input process", OR Spektrum 5, 207-218 (1983).

[8] R.W. Wolf, "Poisson arrivals see time averages", Oper. Res. 30, 223-231 (1982). 